\title{
LETHAL GENES IN INBRED LINES
}

\author{
J. H. BENNETT \\ Department of Genetics, University of Cambridge
}

Received 29.vii.1955

\section{INTRODUCTION}

IsoLATED lethal mutants are of course rapidly eliminated from inbred lines but pairs of completely linked recessive lethal genes lying opposed on homologous chromosomes are able to balance each other and enforce heterozygosity at these as well as at other loci with which they are completely linked. Inbreeding will preserve such balanced lethal systems and the inbred organisms, although alike in genetic constitution, will be maintained in the heterozygous condition at a number of loci.

With lethal mutations not infrequently occurring in nature, it is conceivable that balanced lethal systems might occasionally accumulate and spread through the germ plasm of an inbred line. In the present article, we determine the frequency with which balanced lethal systems can be expected to arise as the result of gene mutation in inbred lines. The rate at which almost-balanced systems of closely linked lethal genes are eliminated from inbred lines is also determined.

\section{EXPECTED LIFE OF A RECESSIVE LETHAL GENE IN AN INBRED LINE}

A lethal gene can form part of a balanced lethal system in an inbred line only if, during its lifetime in this line, another lethal mutant occurs in an appropriate part of the germ plasm. We shall therefore consider at first the rate of elimination and the expected life of a single lethal gene in an inbred line.

We shall suppose that heterozygotes for lethals and lethal-free homozygotes are equally viable. Only a single mating is required for the continuation of an inbred line and we shall therefore suppose that all matings give rise to the same number of viable offspring.

\subsection{Selfing}

Viable organisms carrying a single recessive lethal gene $e$ at some locus must possess the genotype $l /+$ where + denotes any non-lethal allele of $l$. With selfing, the probability of occurrence of this genotype is reduced by one-third in each generation. A measure of the rate of elimination of the lethal gene is therefore given by

$$
-\log _{e}(2 / 3)=0.40546 \text {. }
$$


If $u_{n}$ is the frequency of the genotype $l /+$ in the $n$-th generation, the average number of generations required for the elimination of the lethal gene from the line is

$$
\frac{\stackrel{n=1}{n=1}_{\sum_{n=1}^{\infty}\left(u_{n-1}-u_{n}\right)}^{\infty} n\left(u_{n-1}-u_{n}\right)}{n=0}=\stackrel{S}{\infty}_{n=0}\left(u_{n}\right) .
$$

Putting $u_{o}=\mathrm{I}$, we find that a lethal gene will survive under selfing for an average of 3 generations.

\subsection{Sib mating}

Brother-sister matings involving a single lethal gene are of two types, (r) $l /+\times+1+$ and (2) $l /+\times l /+$ according as the lethal gene is carried in only one or both of the mated sibs. Suppose these two mating types occur in the $n$-th generation of sib mating with frequencies $u_{n}$ and $v_{n}$ respectively.

The generation matrix is as follows:

\begin{tabular}{c|cc|} 
& $(\mathrm{x})$ & $(2)$ \\
\hline$(\mathrm{I})^{1}$ & 2 & 4 \\
$(2)^{1}$ & $\mathrm{I}$ & 4 \\
\hline $\begin{array}{c}\text { column } \\
\text { divisor }\end{array}$ & 4 & 9
\end{tabular}

From the characteristic equation,

$$
(2-4 \lambda)(4-9 \lambda)-4=0
$$

we find that the latent roots are

and

$$
\lambda=(\mathrm{I} 7+\sqrt{ } \mathrm{I} 45) / 36=0.8067 \mathrm{I}
$$

$$
\mu=(17-\sqrt{ } 145) / 36=0 \cdot 13773 .
$$

The rate at which sib matings involving a lethal gene are eliminated is thus $-\log _{e}(0.8067 \mathrm{I})=0.21479$. (With parent-offspring mating, the rate of elimination is $-\log _{e}(0 \cdot 84576)=0 \cdot 16572$.) The principal components of frequency ( $c f$. Fisher, 1949, p. 30) corresponding with the latent roots $\lambda$ and $\mu$ are respectively,

$$
\begin{aligned}
\mathrm{L} & =u+(4 \lambda-2) v \\
\mathrm{M} & =u+(4 \mu-2) v
\end{aligned}
$$

and since $\mathrm{L}_{n}=\lambda^{n} \cdot \mathrm{L}_{o}$ and $\mathrm{M}_{n}=\mu^{n} \cdot \mathrm{M}_{o}$, it follows that

$$
\begin{aligned}
& u_{n}=\left[(64+84 \lambda) \lambda^{n} \cdot \mathrm{L}_{o}+(8 \mathrm{I}-\mathrm{r} 8 \lambda) \mu^{n} \cdot \mathrm{M}_{o}\right] / \mathrm{r} 45 \\
& v_{n}=\left[9(-\mathrm{r} 7+36 \lambda) \lambda^{n} \cdot \mathrm{L}_{o}-\mu^{n} \cdot \mathrm{M}_{o}\right] / 290 .
\end{aligned}
$$


The average number of generations for which a mating of type $l /+x+1+$ will be present is $\sum_{n=0}^{\infty}\left(u_{n}\right)$. Putting $u_{o}=\mathrm{I}$ and $v_{o}=0$, i.e. $\mathrm{L}_{o}=\mathrm{M}_{o}=\mathrm{I}$, we find that the average life of a mating of this type is

$$
\frac{\mathrm{I}}{\mathrm{I} 45}\left[\frac{64+\mathrm{I} 8 \lambda}{\mathrm{I}-\lambda}+\frac{\mathrm{I} 8(8 \mathrm{I}-\mathrm{I} 8 \lambda)}{\mathrm{I}+\mathrm{I} 8 \lambda}\right]
$$

or $3 \frac{1}{3}$ generations. In this case, the mating $l /+\times l /+$ will be present on the average for $\mathrm{I} \frac{1}{2}$ generations, since with $\mathrm{L}_{o}=\mathrm{M}_{o}=\mathrm{I}$,

$$
\begin{aligned}
{\stackrel{S}{S}\left(v_{n}\right)}_{n=0} & =\frac{9}{290}(-\mathrm{I} 7+36 \lambda)\left[\frac{\mathrm{I}}{\mathrm{I}-\lambda}-\frac{\mathrm{I} 8}{\mathrm{I}+\mathrm{I} 8 \lambda}\right] \\
& =\mathrm{I} \frac{1}{2} .
\end{aligned}
$$

When once present, however, the mating $l+\times l l+$ will remain on the average for 3 generations since ${\underset{S}{n=0}}_{n}^{\infty}\left(v_{n}\right)=3$ when $u_{o}=0$ and $v_{0}=\mathrm{r}$.

\section{FORMATION OF BALANCED LETHAL SYSTEMS}

\subsection{Selfing}

We have seen that an isolated recessive lethal gene $l$ survives under selfing for an average of 3 generations. Hence, if at a locus showing no crossing-over with that of the lethal $l$, lethal mutants $l^{1}$ occur at the rate $\nu$ per chromosome in each generation, the probability that the genotype $l / l^{1}$ will arise following on the appearance of the lethal gene $l$ must be $3^{\nu}$. All the progeny obtained by selfing individuals of this genotype will also have the genotype $l / l^{1}$.

\subsection{Sib mating}

There are four types of brother-sister mating involving both of two lethal genes $l, l^{1}$ at two completely linked loci.

(I) $l / l^{1} \times+l+$

(2) $l /+\times l^{1} /+$

(3) $l / l^{1} \times l /+$ and $l / l^{1} \times l^{1} /+$

(4) $l / l^{1} \times l / l^{1}$.

We shall suppose that these matings occur with the frequencies $w_{n}$, $x_{n}, y_{n}$ and $z_{n}$ respectively in the $n$-th generation. The generation matrix is as follows :

\begin{tabular}{c|cccc|} 
& (1) & $(2)$ & $(3)$ & $(4)$ \\
\hline$(1)^{1}$ & $\cdot$ & 2 & $\cdot$ & $\cdot$ \\
$(2)^{1}$ & $\mathrm{I}$ & 2 & 2 & $\cdot$ \\
$(3)^{1}$ & $\cdot$ & 4 & 4 & $:$ \\
$(4)^{1}$ & $\cdot$ & $\mathrm{I}$ & $\mathrm{I}$ & $\mathrm{I}$ \\
\hline $\begin{array}{c}\text { column } \\
\text { divisor }\end{array}$ & $\mathrm{I}$ & $\mathrm{I} 6$ & 9 & $\mathrm{I}$ \\
\hline
\end{tabular}

Matings of type (4) are in the balanced condition and can lead only to matings of this same type. There must therefore be a latent root 
of unity in the characteristic equation. The principal component of frequency corresponding with this root is

$$
\mathrm{Q}_{n}=3 w_{n}+6 x_{n}+\mathrm{10} y_{n}+38 z_{n} \text {. }
$$

Since $w_{n}, x_{n}$ and $y_{n}$ each tend to zero with increasing $n$, the limiting frequency of matings of type (4) is

$$
\left(3 w_{o}+6 x_{o}+10 y_{o}+38 z_{0}\right) / 38 \text {. }
$$

We have seen that after the lethal gene $l$ has appeared, matings $l /+\times+/+$ and $l /+\times l /+$ will be present on the average for $3 \frac{1}{3}$ and $I \frac{1}{2}$ generations respectively. Now a mutation to $l^{1}$ occurring in one of the chromosomes bearing a non-lethal allele of $l$ in the mating $l+\times l l+$ will always lead to a mating of type (3). In the mating $l /+x+1+$, such a mutation will lead to a mating of type (2) in two-thirds of cases and to a mating of type ( 1 ) in one-third of cases. Hence, if at some locus completely linked with that of the lethal $l$, lethal mutants $l^{1}$ arise at the rate $\nu$ per chromosome in each generation, the probabilities that matings of types (1), (2) and (3) will arise following on the appearance of the lethal $l$, are $10 v / 3,20 v / 3$ and $3 v$ respectively.

If the initial mating is type (I), then $w_{0}=\mathrm{I}$ and the limiting value of $z_{n}$ as $n$ increases indefinitely is seen to be $3 / 38$. Initial matings of types (2) and (3) lead to limiting values for $z_{n}$ of $6 / 38$ and $10 / 38$ respectively. Hence, the probability that a sib mating will arrive at the balanced lethal condition at these two loci following on the appearance of the lethal gene $l$ is

$$
\begin{aligned}
\nu\left(\frac{10}{3} \times \frac{3}{3^{8}}\right. & \left.+\frac{20}{3} \times \frac{6}{3^{8}}+3 \times \frac{10}{3^{8}}\right) \\
& =4^{0 \nu / 19 .}
\end{aligned}
$$

In brother-sister matings which are in this condition, certain regions of the germ plasm will be kept heterogeneous and if there is no crossing-over in the affected chromosomes, these regions might be of considerable extent. At loci which are incompletely linked to a balanced lethal configuration, homogeneity will ultimately be attained by continued inbreeding but the approach to this limiting condition will be very slow when the linkage is close (see Bartlett and Haldane, 1937). A special case of balanced lethal genes occurs when these are alleles which yield a viable heterozygote ( $c f$. Chesley and Dunn (1936); Hosino (1943)). The presence of balanced lethal alleles at a number of closely linked loci would completely stop the approach to homogeneity in that part of the germ plasm since two cross-overs between any pair of successive affected loci would be required before any increase in homogeneity could be achieved.

With natural mutation rates, the probability (4ov/rg) that the inbred line will arrive at the balanced lethal condition at two specified loci which are completely linked is so extremely small as to be negligible. 
However, if there is no crossing-over in the chromosome concerned, the appropriate value of $v$ will be the frequency of occurrence of lethal mutants throughout the whole chromosome and this may be as much as 1000 times greater than the natural lethal mutation rate at a single locus. In this case, the probability that the inbred line will arrive at the balanced lethal condition in this chromosome, might be as great as one in a hundred.

\section{EXPECTED LIFE OF AN ALMOST-BALANCED LETHAL SYSTEM}

The genetical situation exemplified in Muller's original demonstration in 1918 of a " balanced lethal " stock of Drosophila with beaded wings, where occasional crossing-over was found to take place between the two lethal genes, would seem to be a more likely result of lethal mutation in inbred lines than the situation we have so far considered. The expected life of a system of incompletely linked lethal genes in the absence of further mutation can be calculated as follows.

\section{I Selfing}

Let $l$ and $l^{1}$ denote lethal genes at two distinct loci with recombination fraction $y$. Organisms heterozygous for both lethals may be classified into two genotypic classes, (1) $l+l+l^{1}$ and (2) $l l^{1} /++$. The generation matrix appropriate to selfing is as follows :

\begin{tabular}{c|cc|} 
& $(1)$ & $(2)$ \\
\hline$(1)^{1}$ & $2(1-y)^{2}$ & $2 y^{2}$ \\
$(2)^{1}$ & $2 y^{2}$ & $2(1-y)^{2}$ \\
\hline $\begin{array}{c}\text { column } \\
\text { divisor }\end{array}$ & $2+y^{2}$ & $3-2 y+y^{2}$
\end{tabular}

The characteristic equation is

$$
\begin{aligned}
& 4(1-2 y)\left(1-2 y+2 y^{2}\right)-2 \lambda(1-y)^{2}\left(5-2 y+2 y^{2}\right)+ \\
& \lambda^{2}\left(2+y^{2}\right)\left(3-2 y+y^{2}\right)=0
\end{aligned}
$$

and in terms of the recombination fraction $y$, the dominant latent root takes the following values :

\begin{tabular}{|c|c|c|}
\hline $\begin{array}{c}y \\
\text { per cent. }\end{array}$ & $\begin{array}{c}\text { Dominant } \\
\text { root } \lambda\end{array}$ & $\frac{1}{1-\lambda}$ \\
\hline 0 & $1 \cdot 00000$ & $\infty$ \\
0 & $0 \cdot 99800$ & 500 \\
$0 \cdot 1$ & $0 \cdot 99001$ & $100 \cdot 1$ \\
$0 \cdot 5$ & $0 \cdot 98005$ & $50 \cdot 13$ \\
$1 \cdot 0$ & $0 \cdot 80628$ & $5 \cdot 16$ \\
5 & $0 \cdot 71644$ & $3 \cdot 53$ \\
10 & $0 \cdot 44444$ & $1 \cdot 80$ \\
50 & & \\
\hline
\end{tabular}

This root is greater than two-thirds only when $y$ is less than 17.894 per cent. and so, when $y$ is greater than this, organisms heterozygous 
for both lethals will be eliminated even more rapidly than those with a lethal gene at only one of these loci. However, for two closely linked lethals, i.e. for very small values of $y$, the dominant root is close to unity, being

$$
\lambda=\mathrm{I}-2 y+\frac{1}{2} y^{2}+\mathrm{O}\left(y^{3}\right) .
$$

Such systems of lethals will therefore be eliminated very slowly and may be said to be almost-balanced. The genotype $l+l+l^{1}$, when once present, can be expected to survive $\mathrm{I} /(\mathrm{I}-\lambda)$ generations and when $y$ is small this is approximately $\mathbf{I} /(2 y)$. With one per cent. crossing-over, for example, the genotype $l+l+l^{1}$ will survive for an average of fifty generations.

\subsection{Sib mating}

There are eight types of sib mating involving both of two lethal genes $l, l^{1}$ at two incompletely linked loci :

(I) $l+1+l^{1} \times++1++$

(2) $l l^{1} /++x++/++$

(3) $l+1++\times+l^{1} /++$

(4) $l+1+l^{1} \times l+1++$ and $l+1+l^{1} \times+l^{1} /++$

(5) $l l^{1} /++\times l+1++$ and $l l^{1} /++\times+l^{1} /++$

(6) $l+/+l^{1} \times l+/+l^{1}$

(7) $l+1+l^{1} \times l l^{1} /++$

(8) $l l^{1} /++\times l l^{1} /++$.

The generation matrix is shown in table 1 . In terms of the recombination fraction $y$, the dominant latent root takes the following values:

\begin{tabular}{|c|c|c|}
\hline $\begin{array}{c}y \\
\text { per cent. }\end{array}$ & $\begin{array}{c}\text { Dominant } \\
\text { root } \lambda\end{array}$ & $\frac{1}{I-\lambda}$ \\
\hline & & \\
0 & 0.00000 & $\infty 9761$ \\
0.1 & 418.4 \\
0.5 & 0.98543 & 68.63 \\
1 & 0.97119 & 34.71 \\
5 & 0.86947 & 7.66 \\
10 & 0.77236 & 4.39 \\
50 & 0.65078 & 2.86 \\
\hline
\end{tabular}

This root is greater than 0.80671 , the value of the dominant root obtained in section 2.2 for a single lethal gene and sib mating, only when $y$ is less than about 8 per cent. For very small values of $y$, the dominant root equals $1-2.4 y$, approximately, so that in this case a mating of type (6) when once present, will survive for an average of $\mathbf{r} /(2.4 y)$ generations.

With parent-offspring mating, twelve mating types need to be distinguished. It can be shown that when the recombination frequency is greater than about I I per cent., parent-offspring matings involving both lethal genes are eliminated more rapidly than those with a 
lethal gene at only one locus. For two closely linked loci, the dominant latent root is approximately $\mathrm{I}-\mathrm{I} \cdot 6 y$ so that a mating involving parent and offspring which both have the genotype $l+l+l^{1}$ will survive, when once present, for an average of $\mathrm{I} /(\mathrm{I} \cdot 6 y)$ generations.

A more complete analysis, taking account of the occurrence of fresh lethal mutants at incompletely linked loci, would be rather complex but it is clear from the above considerations that the probability of an inbred line arriving at the almost-balanced condition at a number of closely linked loci as the result of natural mutation must be so extremely small as to be negligible.

TABLE I

\begin{tabular}{|c|c|c|c|c|c|c|c|c|}
\hline & (I) & (2) & (3) & (4) & (5) & (6) & (7) & (8) \\
\hline $\begin{array}{l}(1)^{1} \\
(2)^{1} \\
(3)^{1} \\
(4)^{1} \\
(5)^{1} \\
(6)^{1} \\
(7)^{1} \\
(8)^{1}\end{array}$ & $\begin{array}{c}\dot{y}^{2} \\
2(\dot{x}-y)^{2} \\
4 y(\dot{x}-y) \\
\cdot \\
y^{2}\end{array}$ & $\begin{array}{c}2(1-y)^{2} \\
2 y^{2} \\
4 y(\dot{r}-y) \\
\cdot \\
(\dot{r}-y)^{2}\end{array}$ & $\begin{array}{l}2 \\
4 \\
\text { I }\end{array}$ & $\begin{array}{c}2 y(1-y) \\
2 y^{2} \\
2(1-y) \\
2(1-y)(2-y) \\
2 y(2+y) \\
(1-y)^{2} \\
2 y(1-y) \\
y^{2}\end{array}$ & $\begin{array}{c}2 y(1-y) \\
2(1-y)^{2} \\
2 y \\
2 y(1+y) \\
2(1-y)^{2} \\
y^{2} \\
2 y(1-y) \\
(1-y)^{2}\end{array}$ & $\begin{array}{c}4 y^{2}(1-y)^{2} \\
4 y^{4} \\
8 y^{2}(1-y)^{2} \\
16 y(1-y)^{3} \\
16 y^{3}(1-y) \\
4(1-y)^{4} \\
8 y^{2}(1-y)^{2} \\
4 y^{4}\end{array}$ & $\begin{array}{c}4 y^{2}(\mathrm{I}-y)^{2} \\
4 y^{2}(\mathrm{I}-y)^{2} \\
2\left(\mathrm{I}-2 y+2 y^{2}\right)^{2} \\
8 y(\mathrm{I}-y)\left(\mathrm{I}-2 y+2 y^{2}\right) \\
8 y(\mathrm{I}-y)\left(\mathrm{I}-2 y+2 y^{2}\right) \\
4 y^{2}(\mathrm{I}-y)^{2} \\
8 y^{2}(\mathrm{I}-y)^{2} \\
4 y^{2}(\mathrm{I}-y)^{2}\end{array}$ & $\begin{array}{c}4 y^{2}(1-y)^{2} \\
4(1-y)^{4} \\
8 y^{2}(1-y)^{2} \\
16 y^{3}(1-y) \\
16 y(1-y)^{3} \\
4 y^{4} \\
8 y^{2}(1-y)^{2} \\
4(1-y)^{4}\end{array}$ \\
\hline $\begin{array}{l}\text { olumn } \\
\text { livisor }\end{array}$ & 4 & 4 & I 6 & 9 & 9 & $\left(2+y^{2}\right)^{2}$ & $\left(2+y-y^{2}\right)^{2}$ & $\left.-2 y+y^{2}\right)^{2}$ \\
\hline
\end{tabular}

\section{DISCUSSION}

Gower, Stadler and Johnson (I946) have reported that of 30 flies taken from a stock of Drosophila which had been mated brother-tosister for 37 generations, I 2 contained a recessive lethal gene in the third chromosome and 7 of these had, in addition, a lethal in the second chromosome. The 7 lethals in the second chromosome were said to be identical as were I I of the I 2 lethals in the third chromosome. There is thus no evidence for the existence of balanced lethals in the second chromosome and if balanced lethals were present in the third chromosome, the probability is only 0.006 that as many as I I out of 12 flies chosen at random would have the one kind of lethal gene. Nevertheless, these authors concluded that in inbreeding there is a strong tendency for lethals to collect and balance one another so retarding further progress towards homozygosis.

The above analysis shows that no such tendency should exist in ordinary inbred stock. However, for any extensive region of the germ plasm where crossing-over cannot occur, there will be a slight but definite tendency for lethals to collect and balance one another in inbreeding. We may therefore conclude that if, in the thirtyseventh generation of these authors' inbred line of Drosophila, balanced lethals were in fact present in a region of the germ plasm in which crossing-over in the females was not restricted, the constituent lethal genes were almost certainly present in the original foundation stock 
from which the inbred line was derived. Since it would be most unusual for two completely linked lethal genes to be present in the foundation stock when this is chosen at random from a freely interbreeding population, we are forced to the conclusion that Gower et al. started their experiment with exceptional material.

In the analysis given above we assumed that the heterozygotes for lethals and the lethal-free homozygotes were equally viable. In Drosophila there is some evidence that heterozygotes for lethals are rather less viable than the lethal-free homozygotes (Stern et al., I952). In this case, the probability of an inbred line arriving at the balanced lethal condition would be even smaller than the value at which we have arrived.

\section{SUMMARY}

Although isolated lethal genes are eliminated by inbreeding, completely linked lethal genes may be so arranged that they constitute a balanced lethal system which, on inbreeding, is perpetuated. In regions of the germ plasm so affected, inbreeding does not lead to a decrease in the amount of heterozygosity. The frequency with which balanced lethal systems will be established in inbred lines as the result of mutation is calculated using matrix methods. With natural mutation rates, the frequency of occurrence of balanced lethal systems is negligible and it is inferred that even closely inbred material will readily eliminate the lethal mutants. With higher mutation rates due to mutagens, or in regions of the germ plasm where crossing-over cannot occur, the frequency of occurrence of balanced lethal systems might not be negligible. A basis is provided for determining what intensity of mutation would be too great for the lethal mutants occurring in inbred lines to be eliminated.

\section{REFERENCES}

BARTLETT, M. S., AND HALDANE, J. B. S. 1937. The theory of inbreeding with forced heterozygosis. 7. Genet., 3I, 327 .

CHESLEY, P., AND DUNN, L. c. 1936. The inheritance of taillessness (anury) in the house mouse. Genetics, 2I, 525 .

FISHER, R. A. 1949. The Theory of Inbreeding. Oliver and Boyd, Edinburgh.

GOWER, J. W., STADLER, J., AND JOHNSON, L. E. 1946. On the mechanism of heterosis - the chromosomal or cytoplasmic basis for heterosis in Drosophila melanogaster. Amer. Nat., $80,506$.

Hosino, y. I 943 . Genetical studies of the lady-bird beetle, Harmonia axyridis Pallas. Jap. F. Genet., 19, 258.

MULLER, H. J. 1918. Genetic variability, twin hybrids and constant hybrids, in a case of balanced lethal factors. Genetics, 3, 422 .

STERN, C., CARSON, G., KINST, M., NOVITSKI, E., AND UPHOFF, D. 1952. The viability of heterozygotes for lethals. Genetics, $37,4^{\mathrm{r}} 3$. 